\section{Deutsche Forschungsgemeinschaft macht sich Sorgen um den akademischen Nachwuchs}

Die Deutsche Forschungsgemeinschaft (DFG) ist in eine schwierige Lage geraten. Immer mehr Wissenschaftler erwarten von ihr finanzielle Förderung. Sie kann diese aber aus Finanznot nicht leisten. Ein zweites Programm, das Bundeswissenschaftsminister Jürgen W. Möllemann vor der DFG-Jahresversammlung am 13. Juni in Regensburg bekräftigt hatte, scheint zwar eine Erleichterung zu bringen, doch seine Finanzierung ist höchst ungewiß.

DFG-Präsident Prof. Dr. Hubert Markl klagte darüber, daß zum Beispiel die Zahl der an jeweils einem Ort bestimmte Themen langfristig bearbeitenden biowissenschaftlichen Forschergruppen ( 21 von insgesamt 33 ) eingeschränkt werden mußte und daß es nicht möglich sei, zusätzlich zu den insgesamt bald sechs Klinischen Forschergruppen weitere derartige vorbildliche Einrichtungen zu schaffen. Hervorragende Forscher drängen aber auf multizentrische Forschergruppen.

Die DFG erhält 1989 vom Bund und den Ländern über 1,1 Mrd DM. Um vor allem dem wissenschaftlichen Nachwuchs zu helfen, brauche sie nächstes Jahr 6,5\% mehr, forderte Markl. Es sei dramatisch, daß die Gutachter mit insgesamt rund einem Drittel viel zu viele der Förderanträge ablehnen müßten. Sie könnten nicht mehr wissenschaftlich urteilen, sondern seien an ein „Quotenspalier" gefesselt.

Nach dem Programm „Möllemann II" sollen Bund und Länder bis zum Jahr 2000 mit insgesamt rund 6 Mrd DM zusätzlich zu den rund 50000 jetzt vorhandenen weitere 10000 neue Stellen für Nachwuchsforscherinnen und -forscher über die DFG und die Max-Planck-Gesellschaft schaffen. Für Markl ist es besonders wichtig, die Nachwuchswissenschaftler jetzt zu fördern. Ihre Zahl dürfte sich in den nächsten sechs Jahren noch um etwa $40 \%$ erhöhen. Nachher sei aus demographischen Gründen mit geringeren Jahrgangsstärken zu rechnen.

Die DFG fördert rund 4700 Einzelvorhaben im Normalverfahren, 118 überregionale Schwerpunkte, 33 Forschergruppen und 156 Sonderforschungsbereiche. Mehr als ein Drittel der Mittel entfällt auf die Biowissenschaften, die Hälfte davon (180 Mio DM) auf die Medizin und die Ernährungswissenschaften. Die Misere der DFG rührt daher, daß die Zahl der antragstellenden Forscher in den letzten zehn Jahren um $50 \%$ (auf rund 15000) gestiegen ist, ihre Mittel von Bund und Ländern aber nur um $5 \%$.

Eine neue Epoche der Organtransplantation skizzierte Prof. Dr. Rudolf Pichlmayr von der Medizinischen Hochschule Hannover vor der DFG. Immunologische Toleranz des empfangenden Organismus kann danach auf zwei Wegen erreicht werden. Mit Hilfe monoklonaler Antikörper könnten
Interleukin-2-Rezeptoren ausgeschaltet werden, die in einer bestimmten Phase von den T-Lymphozyten exprimiert werden, welche die Antigene erkennen. Daraus entsteht ein spezifischer immunologischer Defekt, die Zellen werden gegenüber einem bestimmten Antigen tolerant. Neben diesem Konzept der phasenbezogenen Toleranzerzeugung wurde in letzter Zeit auch eine gentechnologische Methode entwikkelt: Körpereigene Zellen werden transfiziert, so daß sie ein bestimmtes fremdes Antigen selbst exprimieren, also tolerogen wirken. Experimente haben nun gezeigt, daß die Immunreaktion auch gegen andere auf derselben Transplantatzelle sitzende Antigene stattfinden kann. Das wird vor allem mit der Induktion von Suppressorzellen erklärt. Würden diese zunächst vor allem in der Grundlagenforschung entwickelten Konzepte in der Klinik realisiert, wären nach Pichlmayr die Hauptprobleme einer Abstoßung oder einer Infektion durch Immunsuppression beseitigt. Er hält es auch für möglich, daß Organsysteme wie Lunge, Pankreas, Haut und andere erfolgreich verpflanzt werden können, ja sogar die Übertragung von Organen tierischer Herkunft auf den Menschen wäre mit geringem Risiko möglich. Voraussetzung für solche Entwicklungen ist aber die enge Zusammenarbeit von Grundlagen- und klinischer Forschung. Der Hannoveraner Wissenschaftler bestärkte die in der Ärzteschaft nicht unumstrittene Forderung des Wissenschaftsrates und der DFG, daß mehr Naturwissenschaftler und experimentell-theoretisch Erfahrene in oder mit den Kliniken arbeiten müßten und daß der klinisch Tätige an fundierten, auch theoretisch-experimentellen Forschungen teilhaben müsse. Für nicht-klinische Fachleute müßten allerdings befriedigende Lebenspositionen geschaffen werden.

\section{Max-Planck-Gesellschaft: Regionales Gefälle in Europa beseitigen}

Forschungspartikularismus und Ungleichgewichte in der Wissenschaft machen der Max-Planck-Gesellschaft (MPG) Sorgen. Die zunehmenden Egoismen der Bundesländer betreffen sie unmittelbar, wenn diese das jetzt 40 Jahre alte Instrument der Gemeinschaftsfinanzierung mißbrauchen und auf einem „just return“ der Mittel bestehen, die sie dafür ausgeben. Dies werde dem Geist des Solidaritätspaktes nicht mehr gerecht, tadelte Präsident Prof. Dr. Heinz A. Staab auf der diesjährigen MPG-Jahresversammlung am 9. Juni in Wiesbaden: „Es wäre verhängnisvoll, wenn die gemeinschaftliche und arbeitsteilige Förderung der überregional bedeutsamen Forschungseinrichtungen wie etwa der MaxPlanck-Gesellschaft oder der Deutschen Forschungsgemeinschaft durch ein enges provinzielles Denken, das vor 40 Jahren die erste Politiker-Generation der Bundesrepublik ausdrücklich vermeiden wollte, in Gefahr geriete." 